relations institutionnelles afin de sensibiliser, de mobiliser et d'associer l'ensemble du Mouvement Desjardins aux projets menés par la SDID. Également, elle souhaitait favoriser le développement de partenariats et aider l'accroissement de la présence de la société dans les domaines de l'éducation et du développement.

Le nouveau millénaire débute sur un ton joyeux alors que DID célèbre ses 30 ans d'existence et le Mouvement Desjardins fête son centenaire. Une belle occasion de faire connaitre le Mouvement Desjardins aux collaborateurs du DID notamment par une série d'événements promotionnels, de séminaires et de publications. En 2000, l'association de trois filiales du Mouvement mènera à la création du Fond d'investissement pour le développement international (FONIDI). Ce levier financier bénéficie à la croissance d'institutions coopératives, mutualistes ou de finance de proximité. Les campagnes promotionnelles passent, de plus en plus, par la valorisation du site Internet. Les outils de communications sont variés et tendent à rejoindre différents publics.

Les valeurs et principes fondamentaux de DID sont au cour des messages de communication institutionnelle de l'organisation depuis sa création. Ils sont davantage véhiculés depuis les dernières années. C'est pourquoi, DID place les individus au centre de ses actions. Elle promeut donc une des principales valeurs du Mouvement Desjardins, l'argent au service des gens. Un survol de la stratégie de communication est opéré en identifiant les éléments qui la définissent d'abord dans une logique d'association puis évoluant vers une logique d'entreprise.

Le second chapitre traite de la communication interne et externe par l'explication et l'analyse de ses composantes. D'abord, les premiers publics visés comme l'ACDI et le Mouvement Desjardins puis se dirige vers les partenaires et les bénéficiaires locaux. À cela, s'en suivra la valorisation de la communication interne en passant par les employés de DID puis s'étendra aux bailleurs de fonds et bien d'autres intervenants. Les principaux canaux de communication sont relevés et décrits ainsi que l'évolution des moyens de communication commençant par des rencontres personnalisées pour se tourner davantage vers l'organi- sation d'événements et l'utilisation des médias électroniques.

Cet ouvrage trace la ligne du temps parcouru par DID de sa création à aujourd'hui. Tout au long de sa sinueuse trajectoire, la filiale de Desjardins apprend, découvre et s'adapte à son univers de travail qu'est l'étranger. On y met en valeur une philosophie de gestion qui privilégie l'esprit d'innovation et place le développement au sein des communautés moins nantis au centre de ces priorités. Le désir d'atteindre ses objectifs jumelés à la force de partenaires marquants comme l'Agence canadienne de développement international (ACDI) ont menés DID à des sommets inégalés.

L'auteure nous introduit activement dans le Mouvement Desjardins et nous permet de découvrir une filiale unique, laquelle a su se positionner et évoluer au rythme des décennies. Il est intéressant de voir à quel point les moyens et les messages de communication ont réussi à atteindre les publics visés, à les informer et à les intéresser sur les activités de DID. Nous pouvons tirer de belles conclusions de cette aventure dans le monde de la microfinance local et international.

Marie-Anne Blackburn, M.B.A.

Candidate au doctorat, Université d'ÉvryVal d'Essonne

Chargée de projets pour le Bureau international Université du Québec à Chicoutimi

\section{Douesnard, J. (2012). La santé psychologique des pompiers, Québec, Presses de l'Université du Québec, 108 p.}

Le livre de Jacinthe Douesnard présente le compte-rendu d'une étude sur différents aspects de la santé psychologique des pompiers : détresse, épuisement, stress post-traumatique, problèmes de sommeil et état de santé général, notamment. Les résultats de son enquête sont mis en contexte par une présentation succincte mais très informative de la psychodynamique du travail. L'auteur développe deux aspects importants du vécu des pompiers et des stratégies d'adaptation au danger: la soumission aux 
règles de l'équipe d'intervention et les réactions ambivalentes face au feu, soit l'attrait et la peur. Le premier aspect est étroitement associé au collectif de travail: la prise de décision, l'entraide et la reconnaissance sont au cœur de l'exercice du rôle de pompier. Il n'est pas possible d'envisager le combat contre les incendies en solitaire : le partage des risques, la coordination des efforts et la solidarité dans l'action caractérisent le métier. Il en découle une solidarité identitaire chez les pompiers.

L'autre aspect est celui du combat: le feu constitue un ennemi contre lequel il faut se préparer, s'unir et surmonter ses peurs si on aspire à la victoire. La montée d'adrénaline liée au danger provoque conjointement la peur et l'excitation. La crainte d'être exposé à la souffrance d'un enfant ou d'un proche, d'être incapable d'intervenir ou d'être exposé à des produits dangereux suscite l'angoisse, d'une part, mais l'excitation du combat à mener, de la sollicitation des ressources physiques et psychiques, de la possibilité d'avoir à manifester de l'héroïsme pour secourir une victime provoque l'exaltation, d'autre part. L'attente à la caserne définit également la réalité du pompier : d'un côté, la camaraderie, le deuxième chez-soi, la famille professionnelle, mais de l'autre côté, l'ennui et la promiscuité sont la source d'un autre type de stress. Les pompiers aimeraient être plus souvent sur le champ de bataille plutôt que d'attendre les alertes.

Plusieurs données de l'enquête sont surprenantes: 1) il y a moins de cas de détresse psychologique chez les pompiers que dans l'ensemble des travailleurs, 2) la détresse psychologique rapportée est moins forte, 3) l'épuisement professionnel et les cas de stress post-traumatique sont relativement rares et 4) l'état de santé général des pompiers est meilleur que celui des préposés aux appels d'urgence 9-1-1. Finalement, les pompiers en situation de grande détresse psychologique préfèrent $(60 \%$ des réponses) recourir à des aidants naturels (conjointe, ami, famille) qu'à des collègues, au supérieur immédiat ou au programme d'aide $(30 \%)$. Les pompiers passent tant de temps ensemble qu'il leur est possible de discuter de nombreuses situations et de connaître l'avis des collègues. Il est possiblement plus facile et fréquent d'aborder les faits d'armes que les vulnérabilités avec ces personnes, surtout dans un contexte d'interdépendance si grands face aux dangers. Cette résistance des pompiers aux problèmes psychologique a d'autres sources comme l'entrainement, la préparation, le soutien de l'équipe et la reconnaissance sociale.

\section{Stéphane Aubin, D. Sc. (gestion), Professeur Université du Québec à Chicoutimi}

\section{Hernandez, É.-M. (2010). L'entrepreneur : Une approche par les compétences. Cormeilles-le-Royal, Éditions EMS, Management \& Société, 224 p.}

C'est le quatrième ouvrage du professeur Hernandez que j'ai l'opportunité de recenser dont son magistral L'entrepreneuriat : Approche stratégique (le compte-rendu fut publié dans ces pages). Oublions le sous-titre, pour autant que je sache un tant soit peu ce qu'est l'approche par les compétences, il me semble avoir été ajouté faute d'imaginer un sous-titre plus approprié. En effet, Revue de la littérature aurait été préférable mais ne fait pas très accrocheur. Or, pourtant, c'est bien ce dont il s'agit ici, et, à mon avis, c'est qui fait l'intérêt de cet ouvrage où la grande érudition de l'auteur permet d'offrir un vaste ensemble des contributions ayant trait à l'entrepreneuriat comme en fait foi une bibliographie de plus de treize pages.

Malgré les réserves exprimées sur le sous-titre, que l'on ne se méprenne pas: il est ici beaucoup question des compétences de l'entrepreneur. N'entreprend pas qui veut mais qui peut. Alors qu'en Beauce on vient d'initier une formation pour futurs entrepreneurs, Hernandez soulève la question maintes fois formulée: la compétence entrepreneuriale relève-t-elle de l'inné ou de l'acquis? En d'autres mots: est-il possible de former des entrepreneurs? Pour l'auteur, l'acquis l'emporte sur l'inné, en conséquence il estime qu'une pédagogie de l'entrepreneuriat trouve ainsi sa signification et son utilité. Une citation de Peter Drucker datant de 1985 sert d'appui à 